\title{
Ein neues absolut messendes Coulometer zum Bestimmen von Chlorid')
}

\author{
Von W. LANGi und K. BORNER \\ Aus dom Instilut fur Kliniscbe C'bemie und Klinische Biochomiv (Dircklor: Prof. Dr. H.-J. Dulc.o), \\ Klinikerm Stoglitz dor IFreien Universität Bcrlin
}

(I3ingegangen ain 9. August 1971)

Jis wird ein neucr Chloricl-Ionen-'Sltrator beschrielsen. Das Gerät arbeitet nach dem veon Cortoves angegebenen Prinæip der Coulonnetrie mil amperometrischer Jändpunktbestimmung. Dic neu ent wickelte Lilektronik des Gerötes ermïglicht Absolutmessungen in Milli-Coulomb oder mMol/J. Das Gerät kann direkt an cinen Computer angeschlossen werden. Jis \%cielnnet sich durch einfache J3edienung und herhe Zuvcrlïssigkeit aus.

Erste praktische lirfahrungen bel der 'Titration von Chlorid bestïtgen die in die Neukonstruktion gesetzen liswartungen.

\section{A nen absolute coulomelor for lbe dotermination of cbloride}

$\Lambda$ new chloride ion titrntor is described. 'The apparatus works on the Cortoovu principle of coulonletry with amperemetric end point determination. Neivly developed electronics are incorporated, which permit alssolute mensurements in milli-coulombs or mmol/l. J'he apparatus can be linked directly to a computer. It is simple to operate and very reliable. Preliminary results from the titration of chloride show that the performance of the new apparatus meets all expectations.

Dic 'l'ittation vón Chlorid durch cine Silberelektrode und Bestimmen des Endpunktes durch Messen der l.eitfähigkcit gilt als cine der «uverlässigsten klinischchemischen Routine-Methoden (1). Dic \%ur "Zeit marktüblichen Geräte, dic zumcist nach den ursprünglichen Vorschlägen von Co'rtove (1) gelsaut sind, eignen sich nicht ohne Umbau für den On-line-Betricb mit cinem Computer. In der Arbeitsweise nach der Originalvorschrift wird das Gerät durch Analyse einer Jïchlösung geeicht. Dicse Eichung $\mathrm{mu} / 3$ in regelmäßigen $\Lambda$ bständen wiederholt werden, um die Veränderungen an der Generator-Elektrode zu berücksichtigen. Eine Vollmechanisicrung ist bei dicsem Verfahren nicht möglich. Dic vorlicgende Arbeit beschreibt cinen Chlorid-Titrator mit stark veränderter Elcktronik. Er erlaubt Absolutmessungen in MilliCoulomb und Milliäquivalent, entsprechend in Mol pro Liter, und erfüllt so eine wichtige Voraussetyung für dic Vollmechanisierung. Úber cine Kopplungselektronik (intcrface) kann das Gcrät an cinen Rechncr angeschlossen werden. Die Konstruktion des Gerätes und erste praktische Erfahrungen werden beschrieben.

\section{Reagenzien}

0,1 M Natriumchlorid-Ejchlösung (7ijtrisol/Mcrck).

Jisessig-Salpetcrshure-Rengen\%: $6,4 \mathrm{ml}$ kon\%. Silpctursihurc p. a. $+100 \mathrm{ml}$ Eiscssig, dest. Wasser ad $1 \mathrm{l}$.

Gelntinc-Jösung: $6,2 \mathrm{~g}$ Gelatine ad 11 dest. Wasscr.

\section{Mesansalz:}

$3,00 \mathrm{ml}$ Risessig-Salpctersäure-Rengenz.

$0,2 \mathrm{ml} \mathrm{Golatinc-Lösung.}$

$0,050 \mathrm{ml}$ Prolsc bzw. läichlösung.

1) Mit Unterstïtzung des Bundesministers fiir Bildung und Wissenschaft (Projekt DV 5305).
Zu Beginn der Messungen wird init ciner lïichlösung der Rengenzien-l,eerwert 3 mal bestimme, der bei dom beschriebunen Gerät etwa 17-21 mMel/l beträgt. Dor l.ecrwert wird an Gerït cingestellt und bei den nachfolgenden Messungen automatisch alogezoggen.

liinzellheiten s. Jjedienung.

\section{Beschreibung des Gerätes}

\section{Allgameine Bescljribung der. Elek:ronite}

Das Meßprinzip des Chlorid-'Titrators beruht auf $\mathrm{dem}$ 1. Faradiy'schen Gesetz:

$$
\text { n }: \frac{1}{1 i} \cdot \mathrm{i} \cdot \mathrm{t}
$$

$n=$ Jadlungsmenge

$F=$ liaraday - Konstante $=96520[\mathrm{As} / \mathrm{Mol}$ Elcktronen $]$

$\mathrm{i}=\mathrm{Strom}[\mathrm{A} \mid$

$\mathrm{t}=\mathrm{kcit}[\mathrm{s}]$.

liür cinc cinwandfreic Bestimmung von $n$ muß in dicser Darstellung cin alssolut konstanter Strom (unabhängig von Temperaturcinflüssen, Zustand der Ej)cktroden und Anfangsleitfähigkcit: der Probc) crzeugt und cinc genaue "zcitmessung vorgenommon werclen. Eine Unabhängigkeit von diescn Fehlern kann durch Bilden des "Zcitintegrals über den Strom errcicht werden. Da für das Meßgcräit cine digitale Anzeige angestrebt wurde und lntegrationen mit großen Zeitkonstanten in der Jilektronik Schwierigkeiten berciten, empfichlt: sich eine Integration des Meßcrgebnisses in klcinen liin\%clschritten.

Bei der Dimensionlerung des Integrierers wurde von folgenden Uberlegungen ausgrgegangen:

Der optimale Me(3bereich des Gerittes sollte bei etwa $100 \mathrm{mMol} / 1$ (hlorid liegen. Dalsei wird eine Auflösung von etwn $0,1 \%$ angestrclut. Bui cincer Gesnmtmeßzeit 
von 20 Sek. und einem Probenvolumen von $50 \mu \mathrm{l}$ errechnet sich für die genannte Konzentration ein Generatorstrom $\mathrm{i}$ :

$$
\begin{aligned}
\mathrm{i} & =\frac{\mathrm{c} \cdot \mathrm{v} \cdot \mathrm{F}}{\mathrm{t}}=\frac{0,1 \cdot 0,05 \cdot 10^{-3} \cdot 96520}{20} \\
& =\underline{\underline{24,13 \mathrm{~mA}}}
\end{aligned}
$$

Durch Ändern des Stromes auf 25,0 mA und Unterdrücken jedes zweiten Zählimpulses kann man die umgesetzte Ladung bei gleichem Probenvolumen direkt in $\mathrm{mCb}$ messen.

Die Funktion des Gerätes wird durch Block- und Impuls-Diagramm beschrieben (Abb. 1 und Abb. 2).t In das Reaktionsgefä $\beta$ ragen zwei Elektrodenpaare, die von galvanisch getrennten Stromkreisen gespeist werden. Die Indikatorelektroden dienen zur Leitfähigkeitsmessung. Die angeschlossene Komparatorschaltung hat einen niedrigen Schwellwert zur Signalisation des Titrationsbeginns mit einer Anzeigelampe und schaltet dann auf einen höheren Schwellwert um, der dem Abschaltpunkt der Titration entspricht. Durch die Generatorelektroden fließt ein konstanter Strom und wird jeweils von einem festen Anfangswert aus über kleine Zeiteinheiten integriert. Der auf den Integrierer folgende Komparator bewirkt die Quantelung und erzeugt je einen Zählimpuls bei Erreichen der Komparatorschwelle. Über eine vom Indikator gesteuerte Torschaltung gelangen die Zählimpulse über die Umschalteinheit ( $\mathrm{mMol} / 1$ oder $\mathrm{mCb}$ ) auf den dekadischen Zähler und die Zifferanzeigeeinheit. Der bei jeder Messung zu berücksichtigende Leerwert der Reagenzien wird an einem dreistelligen Codierschalter einmal vorweg eingestellt. Beim Beginn der Titration wird der Zähler auf diesen Wert automatisch zurückgestellt, der dann vom Meßergebnis subtrahiert wird. Am Endpunkt der Titration wird der Indikatorschwellwert überschritten und damit der Zählvorgang unterbrochen. Gleichzeitig werden Generatorstrom und Rührer abgeschaltet und der Zählerstand wird vom Interface, das nur zur Anpassung an den Rechner dient, übernommen. Ein zeitlich verschobener Interrupt-Impuls signalisiert dem Rechner den bis zum nächsten Start anstehenden Meßwert.
Abb. 1

Blockdiagramm des ChloridTitrators
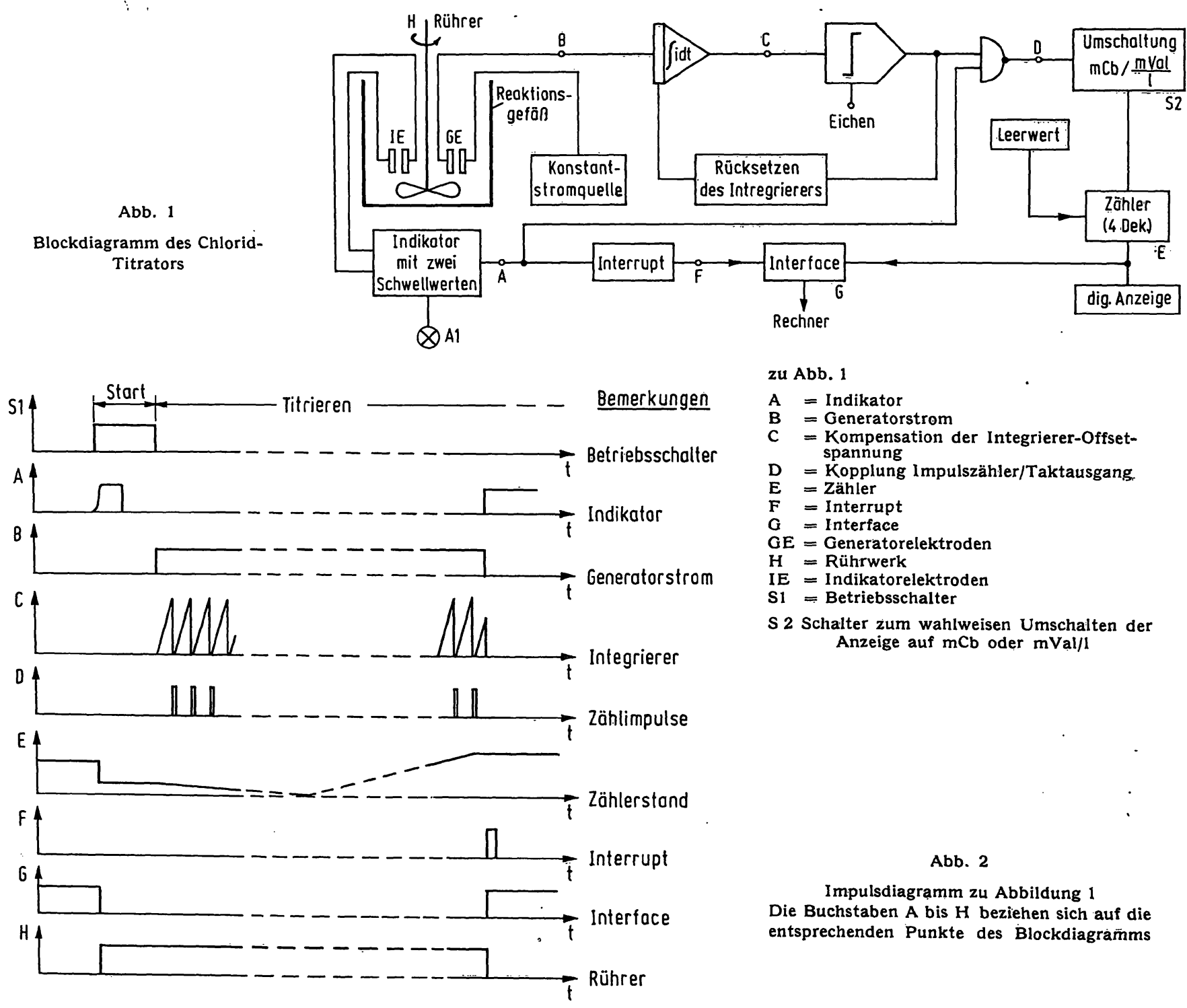

zu Abb. 1

$A=$ Indikator

B = Generatorstrom

C = Kompensation der Integrierer-Offsetspannung

D = Kopplung Impulszähler/Taktausgang

$\mathrm{E}=$ Zähler

$\mathrm{F}$ = Interrupt

$G=$ Interface

$\mathrm{GE}=$ Generatorelektroden

$\mathrm{H}=$ Rührwerk

$\mathrm{IE}=$ Indikatorelektroden

$\mathrm{S} 1$ = Betriebsschalter

S 2 Schalter zum wahlweisen Umschalten der Anzeige auf $\mathrm{mCb}$ oder $\mathrm{mVal} /$

Abb. 2

Impulsdiagramm zu Abbildung 1 Die Buchstaben A bis $H$ beziehen sich auf die entsprechenden Punkte des Blockdiagramms 
Für den Versuchsaufbau des Gerätes wurden von einem marktüblichen Chlorid-Titrator ${ }^{2}$ ) die Elektroden und das Rührwerk übernommen. Die gesamte Elektronik, einschließlich der Netzteile, ist auf drei Steckkarten im Europa-Format aufgebaut. Als Anzeigeeinheit wurde ein Anzeigeblock mit 4 Ziffernanzeigeröhren und drei Codierschaltern zur Einstellung des Leerwertes vorgesehen ${ }^{3}$ ).

\section{Herstellungskosten}

Der Materialwert der Elektronik und der mechanischen Einzelteile beträgt etwa $850,-\mathrm{DM}$ ausschließlich Rechner-Interface (Dezember 1970).

\section{Eicbung der Elektronik}

Die Eichung des Gerätes ist ein einmaliger Vorgang, den der Anwender nicht zu wiederholen braucht. Im Gegensatz zum marktüblichen Titrator, bei dem die Genauigkeit der Eichung von der Eichlösung und dem pipettierten Volumen abhängt, liegt der besondere Vorteil des echt integrierenden Verfahrens in der Absoluteichung. Man benötigt dazu ein digitales Vieltachinstrument der Genauigkeitsklasse 0,1 und einen Frequenzmesser. oder Impulszähler mit einer Meßzeit von 10 Sek. oder mehr. Nach einer ebenfalls nur einmal durchzuführenden Kompensation der IntegriererOffsetspannung (Punkt C, Abb. 1) wird zunächst bei betriebswarmem Gerät der Konstantstromgenerator auf den errechneten Wert von $24,13 \mathrm{~mA}$ eingestellt. Danach wird der Impulszähler an den Taktausgang (Punkt D, Abb. 1) angekoppelt und der auf den Integrierer folgende Komparator so abgeglichen, daß z. B. in 10 Sek. 500 Impulse gezählt werden. Nach diesem Abgleich entspricht ein digit der Anzeige einer Konzentration von $0,1 \mathrm{mMol} / 1$ Chlorid.

Unter der Voraussetzung, daß Integrierer und Komparator eine hohe Langzeitstabilität besitzen, kann nach erfolgter Eichung der zu messende Generatorstrom in weiten Grenzen schwanken, ohne das Meßergebnis $\mathrm{zu}$ beeinflussen. Die Konstantstromquelle kann daher einfacher und preiswerter ausgeführt werden als in bisher üblichen Geräten.

\section{Bedienung}

Zur Messung werden die Elektroden in das Reaktionsgemisch eingeführt. Durch Bedienen des Schalters S 1 (s. Abb. 2) wird die Anzeige auf den am Codierschalter eingestellten Leerwert voreingẹtellt, das Rührwerk eingeschaltet. Die Indikatorlampe leuchtet so lange auf, bis der Indikatorstrom unter einen Schwellwert abgesunken ist. Nach Erlöschen der Lampe wird der Schalter S 1 in die Position "Titrieren" gesetzt. Der Gang der Titration wird fortlaufend angezeigt. Nach Erreichen des Endpunktes bleibt das Rührwerk stehen und die Anzeige bleibt konstant. Damit ist der Arbeitszyklus beendet.

2) Fa. Aminco, bezogen durch Colora-Meßtechnik GmbH.

3) Hersteller: Contraves Industrieprodukte $\mathrm{GmbH}$.

\section{Ergebnisse}

\section{Praktische Erfabrungen}

Die in der Originalmethode (1) vorgeschlagene $\mathrm{Be}$ stimmung des Leerwertes durch Titration einer Lösung, die kein Chlorid enthält, ergibt bei beiden Gerätetypen, dem Originalgerät nach CotLove und unserer Neukonstruktion, einen Leerwert, der 2 bis $3 \mathrm{mMol} / \mathrm{l}$ zu niedrig ist. $\mathrm{Da}$ der Leerwert vom Probenwert abgezogen wird, ist dieser bei der Originalmethode entsprechend etwas zu hoch. Wir empfehlen daher ein anderes Vorgehen:

Es wird eine Lösung mit bekanntem Chloridgehalt wiederholt titriert. Die Differenz aus gefundener und vorgelegter Konzentration ist der Leerwert. Am Gerät (vgl. Abb. 4) wird dieser Leerwert mit Schaltern zu Beginn der Meßserie fest eingestellt. Bei diesem Verfahren ergeben sich im Bereich von 20 bis $200 \mathrm{mMol} / \mathrm{l}$ richtige Ergebnisse in den Grenzen der in Tabelle 1 angegebenen Fehlerbreiten.

\section{Präzision und Ricbtigkeit}

Für wäßr. Lösungen liegt die Präzision in der Serie der Methode zwischen 0,3 und 1,6\%, ausgedrückt als Variationskoeffizient von Wiederholungsmessungen (Tab. 1). Diese Toleranzen sind nur einhaltbar, wenn der Zustand der Generatorelektroden und die Zuverlässigkeit der verwendeten Mikropipette scharf kontrolliert werden. Bei eiweißhaltigen Proben liegt die

Tab. 1

Präzision von Chlorid-Bestimmungen in wäßr. Lösungen (Titrisol, Merck)

\begin{tabular}{ccccc}
\hline $\begin{array}{c}\text { [C1-] vorge- } \\
\text { geben } \\
\text { [mMol/1] }\end{array}$ & $\mathbf{n}$ & $\begin{array}{c}\text { [Cl-] ge- } \\
\text { funden } \\
\text { [mMol/1] }\end{array}$ & $\begin{array}{c}\text { Fehler } \\
\text { [\%] }\end{array}$ & VK [\%] \\
\hline 20 & 8 & 20,2 & $+1,0$ & 8,2 \\
40 & 10 & 40,0 & $\pm 0,1$ & 1,5 \\
60 & 10 & 59,9 & $-0,2$ & 1,8 \\
80 & 10 & 80,1 & $+0,1$ & 0,7 \\
100 & 13 & 100,4 & $+0,4$ & 0,9 \\
120 & 11 & 119,9 & $-0,1$ & 0,8 \\
140 & 9 & 140,0 & $+0,0$ & 0,5 \\
160 & 10 & 159,9 & $-0,0$ & 0,3 \\
180 & 10 & 181,0 & $+0,6$ & 0,6 \\
200 & 10 & 198,9 & $-0,6$ & 0,4 \\
\hline
\end{tabular}

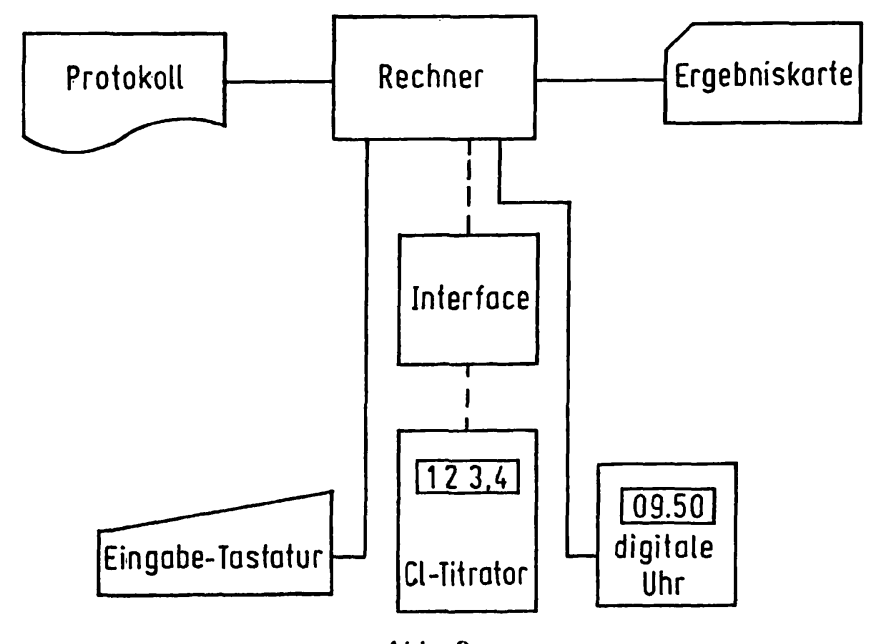

Abb. 3

Verbindung des Chlorid-Titrators mit einem Datenerfassungssystem 


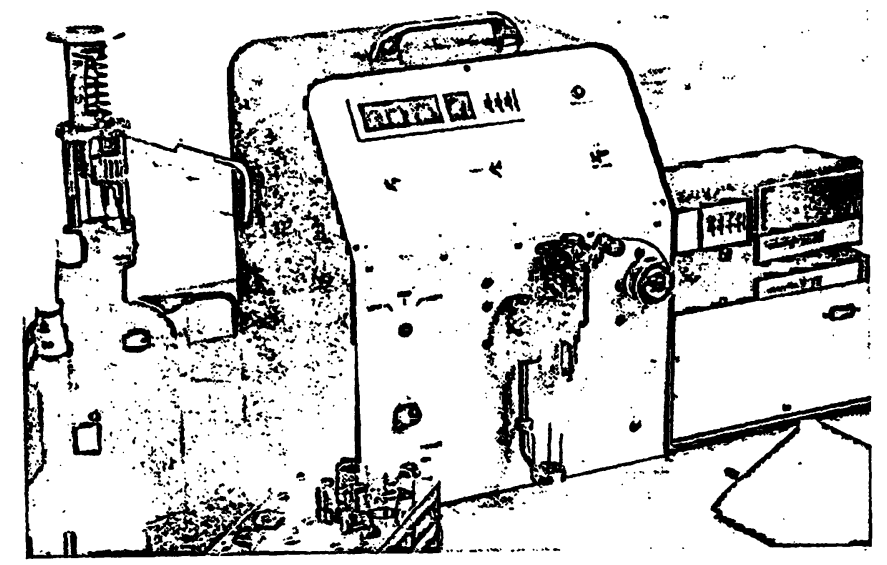

Abb. 4

Der Chlorid-Titrator

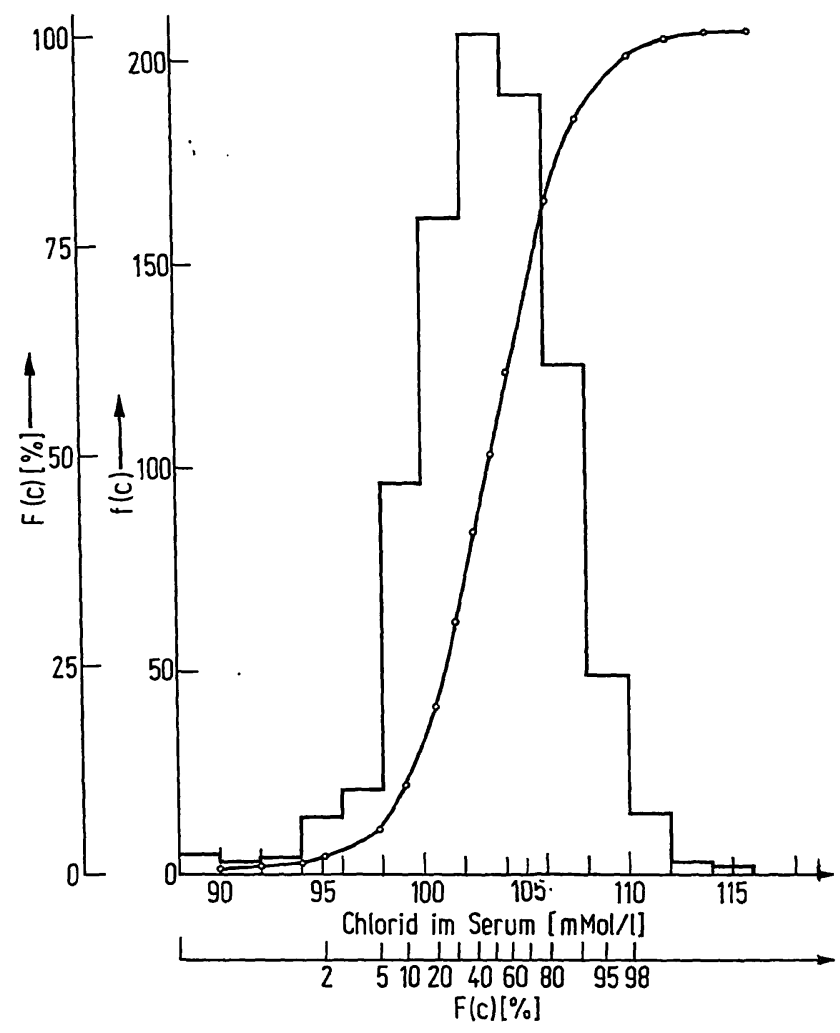

Abb. 5

Serum-Chlorid-Konzentrationen gesunder Erwachsener (18-65 Jahre, $n=895)$. Treppenkurve, relative kumulierte Häufigkeit und Normal-
Präzision in der Serie in vergleichbarem Bereich. So ergab die 20 fache Chlorid-Bestimmung in einer Serie bei einem Rinderserum einen Variationskoeffizienten von $1,6 \%$ bei einer Konzentration von $98,0 \mathrm{mMol} / 1$. Die Bestimmung von Serum-Chlorid-Konzentrationen bei klinisch gesunden Probanden lieferte Werte, die sich mit Literaturangaben decken (Abb. 5). Vergleichsmessungen mit Seren von Normalpersonen an dem Originalgerät nach Cotrove ergaben innerhalb der Fehlerbreite der Methode liegende identische Ergebnisse. $\mathrm{Da}$ das beschriebene Gerät am physikalischchemischen Prinzip der Methode nichts ändert, haben wir auf zusätzliche Versuche zur Richtigkeit verzichtet.

\section{On-line-Betrieb mit einem Datenerfassungssystem}

Abbildung 3 zeigt die Eingliederung des neu entwickelten Gerätes in ein z. Z. iñ Erprobung befindliches Datenerfassungssystem, das die neben dem Meßergebnis zu einem vollständigen Datensatz benötigten Informationen teils automatisch (z. B. Datum und Uhrzeit), teils manuell ( $z$. B. die Patienten-Nummer) erfaßt und weiterverarbeitet. Erste praktische Erfahrungen mit der angegebenen Gerätekombination zeigen einen deutlichen Rationalisierungseffekt im Arbeitsablauf.

\section{Diskussion}

Die von Corrove (1) angeführte Methode der ChloridBestimmung in Körperflüssigkeiten durch Generieren von Silber-Ionen im Reaktionsgemisch gilt allgemein als die einfachste und zuverlässigste Methode (2). Die marktüblichen Chlorid-Titratoren lassen jedoch in bezug auf die Elektronik noch einige Wünsche offen. So sind sie u. a. nicht ohne Umbau an einen Meßrechner anschließbar. Der von uns beschriebene Prototyp ist einfacher $\mathrm{zu}$ bedienen als sein Vorgänger. Durch Wegfall der täglichen Eichung entfällt eine weitere Unsicherheit der Messung. Es sind Absolutmessungen wahlweise in $\mathrm{mCb}$ oder $\mathrm{mMol} / \mathrm{l}$ möglich. Die elektronische digitale Anzeige umfaßt eine zusătzliche Dezimalstelle. Bei Beginn des nächsten Arbeitsganges erfolgt eine automatische Rücksetzung der Anzeige auf den negativen Leerwert. Für den Anschluß eines Computers steht eine Kopplungselektronik (interface) zur Verfügung, die es bisher nicht gab. Das Prinzip der $\mathrm{Ab}$ solutmessung erlaubt theoretisch eine Vollmechanisierung des Verfahrens, die anzustreben wäre.

\section{Literatur}

1. Cotrove, E., H. V. Trautham und R.L.Bowman, J. Laborat.Clin. Med., S. Louis 51, 461 (1958). - 2. Richterich, R., Kli- nische Chemie. 2. Aufl., Akad. Verlags-Ges. Frankfurt (1968) S. 200. 


\section{Auf sicherem Kurs}
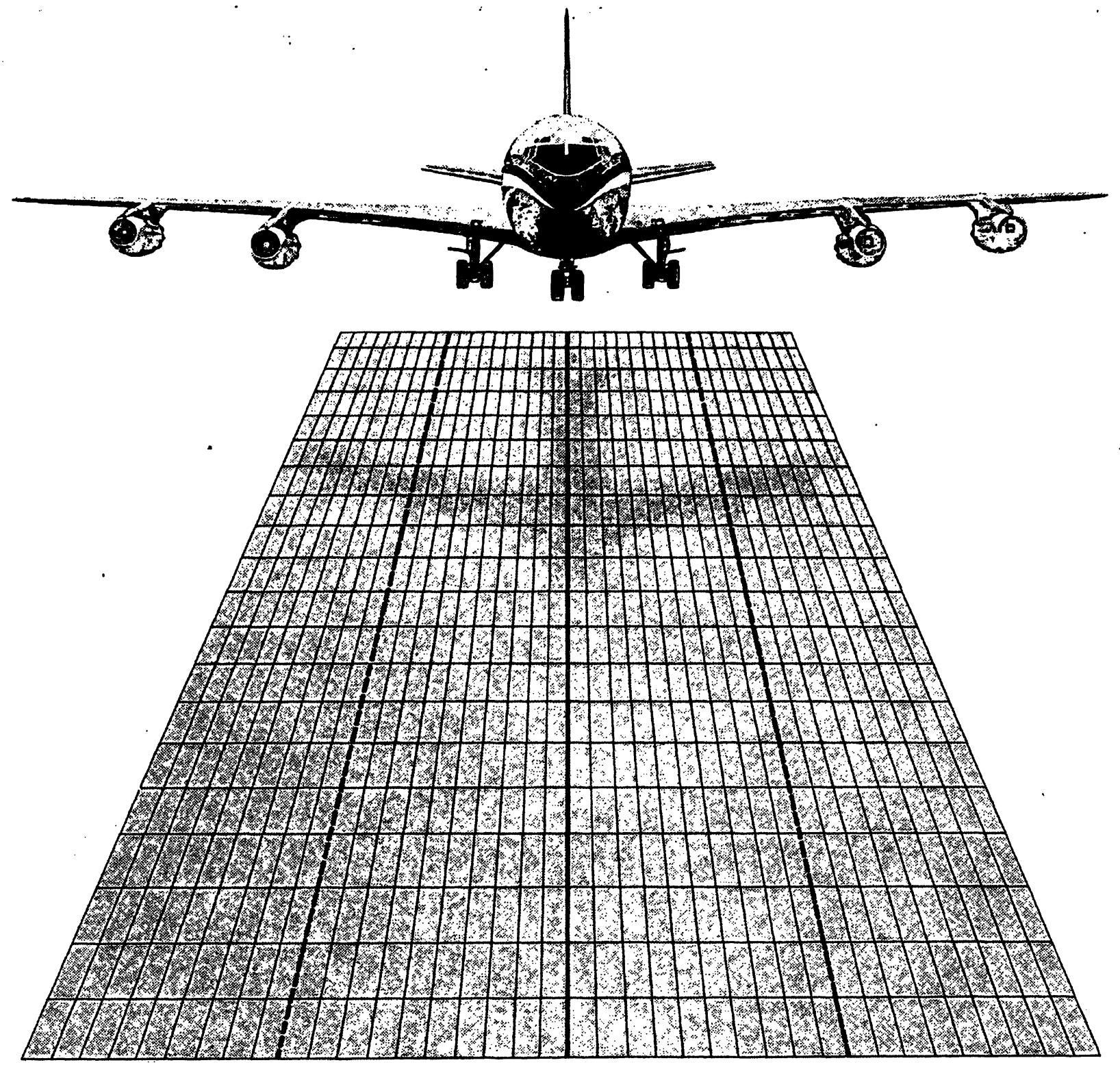

Qualitätskontrolle im klinisch-chemischen Labor mit

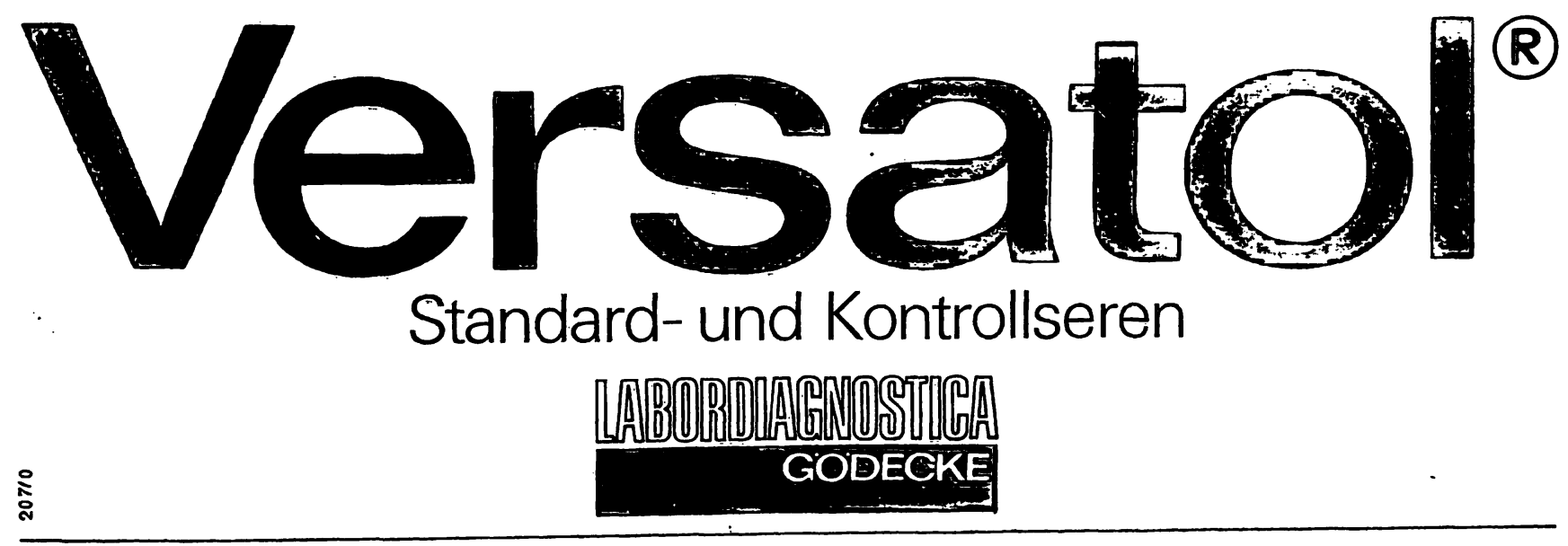


wie PPO, POPOP, Butyl-PBD, TRITON X-100,

Hyamin-Hydroxid usw.

Meßgefäße aus kaliumarmem Glas, Polyäthylen-Meßgefäße, Schraubverschlüsse llefert preiswert:

WERNER ZINSSER SCINTILLATORS

6 Frankfurt 90

Leipziger Straße 65 Telefon 706764

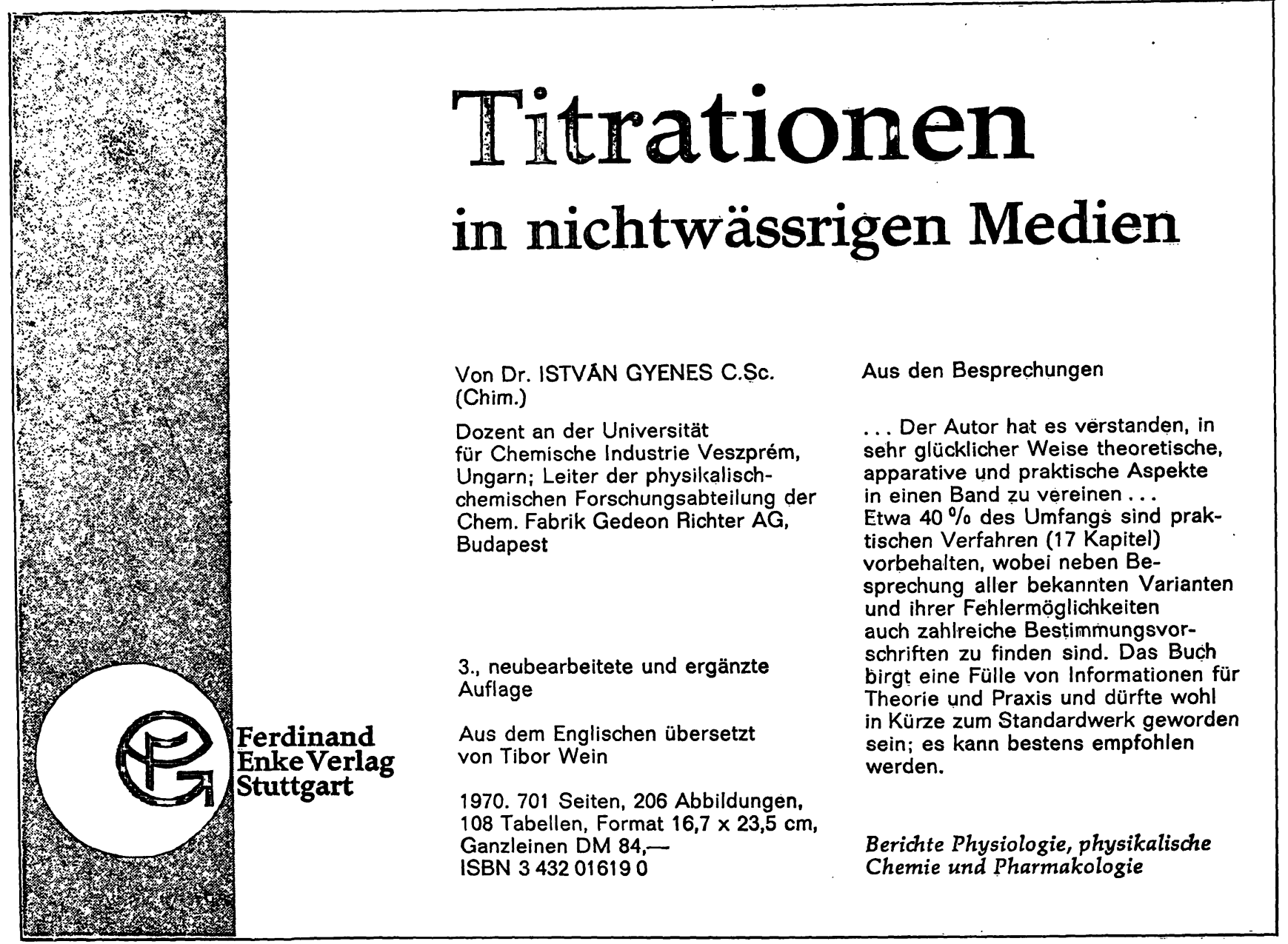

\section{MINI-SCHLAUCHPUMPEN FÜR MEDIZIN, LABOR UND FORSCHUNG}

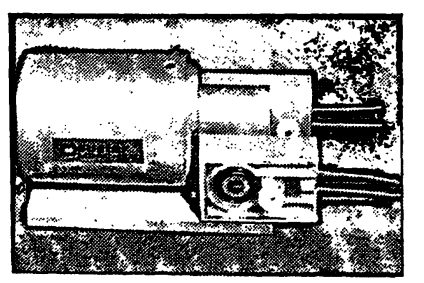

FAVORIT 2000

Förderleistung:

$10-110 \mathrm{ml} / \mathrm{min}$.

je nach Schlauch- $\varnothing$

Maße: $180 \times 100 \times 95 \mathrm{~mm}$

Gewicht: $2,3 \mathrm{~kg}$

Saugleistung: $8 \mathrm{~m}$

Förderhöhe: $25 \mathrm{~m}$

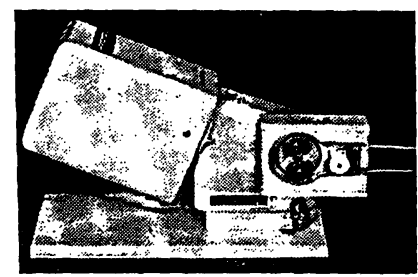

FAVORIT 5000

Stufenlos regelbar

Förderleistung

$0,3-170 \mathrm{ml} / \mathrm{min}$

Maße: $260 \times 100 \times 175 \mathrm{~mm}$

Gewicht: $\quad 3,5 \mathrm{~kg}$

Saugleistung: $8 \mathrm{~m}$

Förderhöhe: $25 \mathrm{~m}$

DE USTE R-Laborgeräte, 8 München 12, Schießstättstr. 27 Tel. 0811/503401 